\title{
Téoros
}

Revue de recherche en tourisme

\section{Le milieu naturel québécois, "c’est beau en grand »!}

\section{Roger Nadeau}

Volume 2, numéro 2, juin 1983

Regards neufs sur les attractions touristiques du Québec

URI : https://id.erudit.org/iderudit/1080834ar

DOI : https://doi.org/10.7202/1080834ar

Aller au sommaire du numéro

Éditeur(s)

Université du Québec à Montréal

ISSN

0712-8657 (imprimé)

1923-2705 (numérique)

Découvrir la revue

Citer cet article

Nadeau, R. (1983). Le milieu naturel québécois, « c’est beau en grand »! Téoros, 2(2), 23-28. https://doi.org/10.7202/1080834ar d'utilisation que vous pouvez consulter en ligne.

https://apropos.erudit.org/fr/usagers/politique-dutilisation/ 


\section{Le milieu naturel québécois, "c'est beau en grand»!}

par Roger Nadeau

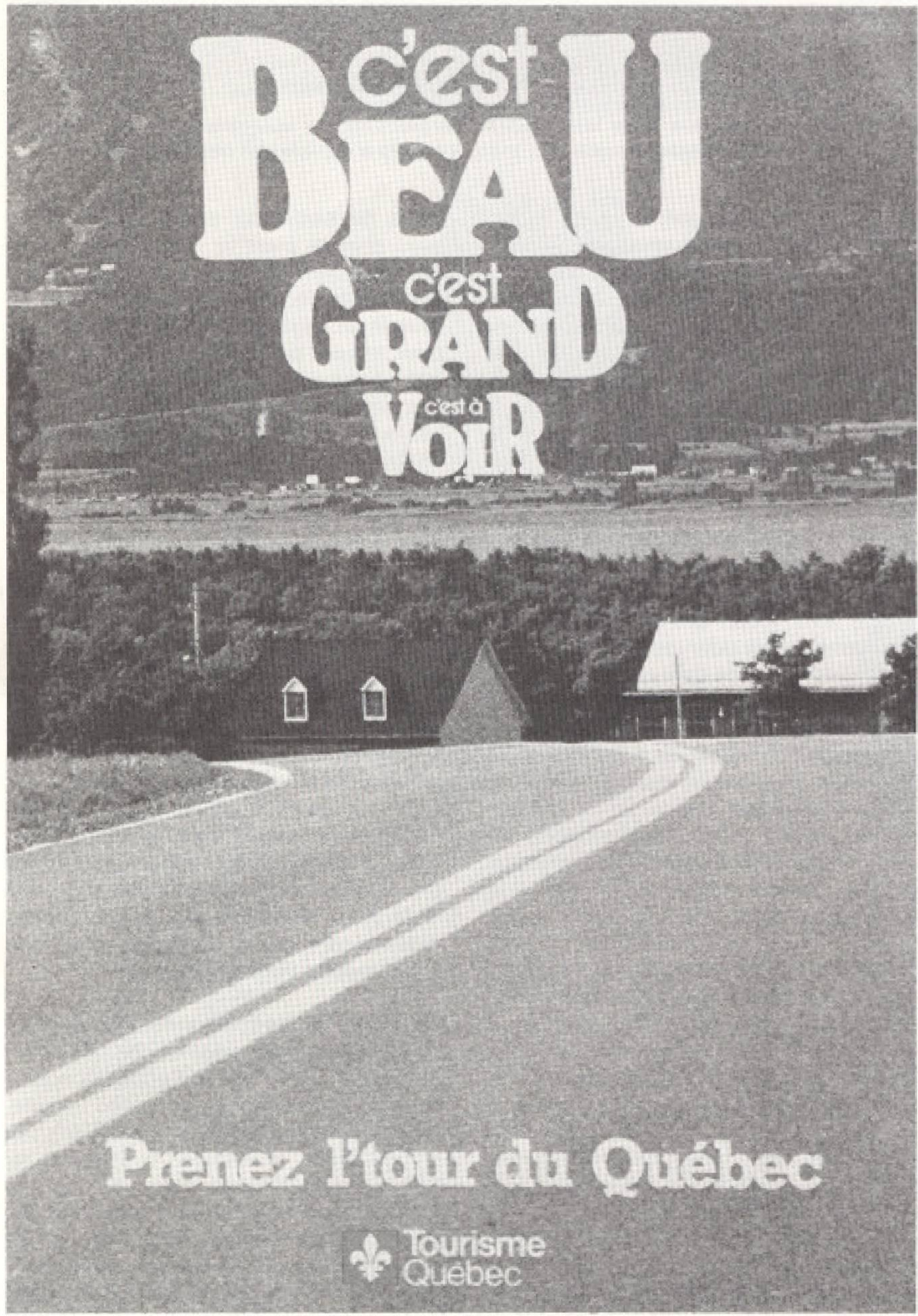

Slogan de la campagne publicitaire de 1981 du ministère de I'Industrie, Commerce et Tourisme.
Depuis fort longtemps, on vante les mérites, les charmes et les attraits du milieu naturel québécois, estimant qu'il s'agit la d'une des principales composantes de notre offre, en plus de représenter un produit remarquable par son originalité et sa spécificité. Déjà, tout jeunes sur nos bancs d'école, nos professeurs nous parlaient avec émotion de la richesse de ce pays au million de lacs poissonneux, aux forêts immenses et giboyeuses, au soussol recélant tellement de trésors abondants que le monde entier en mourait d'envie, à ces paysages uféériquesn qui ont inspiré le poète, le peintre et le chansonnier, à ces montagnes qui permettent à des millions de skieurs de s'adonner à leur sport favori, sans parler de ces grands espaces aux horizons illimités qui s'étendent ả perte de vue et que le pied de l'homme blanc n'a pas encore foulé... II y a 10 ans, 20 ans, ou 50 ans, c'est la vision du Québec que I'on transmettait et c'est celle qu'encore aujoud'hui véhicule le ministère de l'Industrie, du Commerce et du Tourisme avec son slogan: $\alpha c^{\prime} e s t$ beau, c'est grand, c'est à voir.s

Dans le présent article, nous allons tenter, même brièvement, d'analyser un peu ce produit touristique qu'est la nature québécoise; ces considérations, ou plutồt ce wquestionnement» a pour but d"amorcer une réflexion sur la nature de ce produit, sur notre façon de le mettre en valeur et sur l'intérêt qu'il est susceptible de représenter auprès de nos éventuels visiteurs. II y aura donc sans doute plus d'interrogations que de réponses et ce sera aux collègues lecteurs, notamment aux spécialistes de la mise en marché, qu'il reviendra d'apporter leurs éléments de réponse.

Les Québécois dans leur nature

II ne fait pas de doute que le milieu naturel québécois a suffisamment d'attraits pour être un important facteur de déplacement touristique. Bien sôr, il n'y a pas au Québec de canyon du Colorado ni de gorges du Tarn, pas de forêts pétrifiées, ni 
de séquoias de 3000 ans au tronc grand comme une maison, pas de volcans meurtriers et coléreux, ni de gentils petits geysers ayant des facultés curatives reconnues, pas de plages baignées par des mers chaudes, ni de forêtts tropicales où les anthropologues cherchent encore des tribus méchantes et inconnues, pas d'Himalaya, ni de Rocheuses ou d'Alpes, ni de désert à transformer en casino... ou en jardin; Hannibal et ses éléphants n'ont pas eu la brillante idée de faire un détour par Québec, Romus et Romulus auraient pu édifier leur nouvelle civilisation à Sept-Îles plutôt que sur les sept collines de Rome et Louis XIV aurait pu construire Versailles à Bromont ou à Kamouraska. Toutes ces attractions et beaucoup d'autres d'ailleurs attirent des millions de visiteurs. Devonsnous être jaloux de ces arilleurs" et de leurs attractions uspécialesw? Disposonsnous, nous aussi, d'attractions que l'on peut qualifier de abiggest, the largest, the greatest... of the World?" Et puis, tous ces abiggest of the World $\%$, sont-ils bien nécessaires pour intéresser le vacancier à notre produit?

Au Québec, on fait rarement la promotion des divers éléments de notre produit en les qualifiant ainsi par rapport aux autres où qu'ils soient dans le monde, ce qui ne signifie pas qu'on ne pourrait le faire. Par exemple, dans un remarquable livre de I'ATT (Association Technique du Tourisme) portant sur le tourisme québécois (1), on a identifie comme elements de la spécificité québécoise et donc devant susciter un intérêt particulier pour le touriste les aspects suivants: la culture québécoise unique en Amérique et spécialement le fait français; le fleuve SaintLaurent, les grands espaces et l'abondance de la neige. Ces trois derniers éléments représentent des caractéristiques du milieu naturel québécois, ce qui signifie que celui-ci est un facteur extrêmement important sur lequel il faut miser pour accrocher le touriste. II faut alors se demander si cette nature est connue, si elle est mise en valeur, si elle est exploitée selon son potentiel, si elle est rendue accessible, si elle est publicisée, si son caractère de «précieusités lui est reconnu? Bien sûr, on peut relever certains indices qui permettent de dire que certaines clientèles sont deja fort sensibilisées aux beautés et aux ressources de notre environnement. Par exemple, la FAPEL vient de publier quelques données qui sont fort pertinentes pour notre propos en estimant que les villégiateurs du Québec contribuent annuellement $\$ 1,15$ milliards à I'économie de la province. L'organisme rappelle en effet que le Québec compte présentement près de 300,000 résidences secondaires (chalets) et que a/"utilisation $d$ 'un chalet représente en moyenne des retombées économiques annuelles de 3,298\$ ("unite. $n^{(2)}$ C'est au Québec que l'on trouve le plus fort taux de possession de

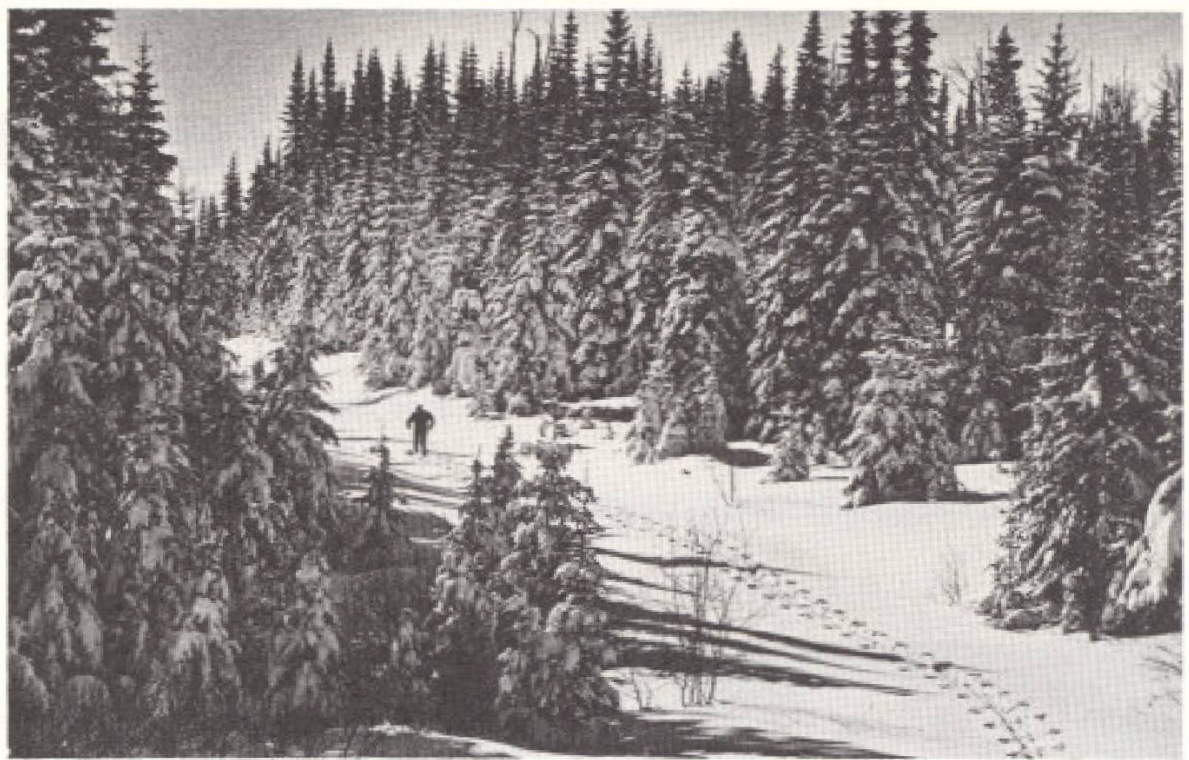

Un nouvel engouement pour les activités de plein-air. La magnificience de la nature québécoise y compte pour beaucoup... mais la nature n'est-elle pas belle partout?

chalet par les ménages; on peut facilement en déduire que la ressource villegiature est à la fois abondante et suffisamment attrayante pour que l'on accepte d'y consacrer des sommes fort rondelettes. On peut sans doute aussi ajouter que l'intérêt que porte les Québécois à la villégiature est une réponse probante à ceux qui s'interrogent pour savoir si la nature, au Québec, représente un attrait touristique.

Mais, il y a d'autres indices: présents lors de la première présentation du Salon Vacances Québec 83 au Complexe Desjardins (avril 1983), des représentants de I'Association des pourvoyeurs du Québec évaluaient à plus de trois-quart de milliard de dollars l'incidence économique liée à leurs actvitiés de chasse et de pêche; l'impact des centaines de milliers d'autres chasseurs et pêcheurs québécois est au moins aussi grand. Encore ici, on admettra qu'il s'agit d'un fort indice en faveur de l'abondance et de la qualité de la ressource. On pourrait apporter encore plein d'exemples allant dans le même sens, allant du nombre d'entrées dans les parcs provinciaux au nombre d'adeptes de ski alpin ou de fond jusqu'au camping d'hiver, aux groupes d'observation des oiseaux et aux clubs de naturistes sereins. Bref, sauf rares exceptions, toutes les données relatives au plein air indiquent une nette progression, un nouvel engouement. C'est sans doute pourquoi $90 \%$ des dépliants promotionnels disponibles lors du récent Salon Vacances Québec 83 visaient a faire connaître des composantes de notre milieu naturel. De telles manifestations sont importantes car elles permettent a ces centaines de milliers de Québécois d'apprendre, d'apprécier ce qu'ils ont chez eux, et avec le temps, on constate que nos compatriotes décou- vrent de nouveaux petits paradis chez eux, comme c'est le cas, par exemple, pour l'archipel de Mingan et ses impressionnantes scultures de calcaire, pour la beauté sauvages des liles de la Madeleine, pour le parc de la Jacques-Cartier, pour nos ressources spéléologiques ou pour des activités unouvelles" comme la descente de rapides en canot pneumatique (Rafting)...

wLe plein air est une façon de vivre que choisit une personne lorsqu'elle explore, découvre, connait et comprend le milieu semi-naturel ou naturel. Le loisir de plein air est donc ce choix que fait une personne lorsqu'elle utilise temporairement l'environnement naturel avec intelligence et connaissance, delicatesse et modestie, détente et plaisir afin de se recréer et de se regénérer. Cette façon de vivre en milieu nature/ implique pour chaque individu une manière d'être, d'agir qui est directement conditionnée par Yenvironnement; elle se réalise pleinement dans les grands espaces caracteristiques suivants: la forêt, la rivière, le lac, la montagne, l'atmosphère, la mer, le milieu souterraín et les milieux intermédiaires. ${ }^{3}$ )

La nature attire-t-elle naturellement? Bref, on pourrait passer des journées entières à vanter les merveilles du milieu naturel québécois et on aurait raison. Mais, I'Ontarien aurait tout aussi raison. Et l'Américain, le Brésilien, le Français, le Suisse ou le Chinois aussi. La nature n'est-elle pas belle partout, voire même exceptionnelle? Les déserts arides et la calotte polaire sont eux aussi plein d'exotisme et d'attraits pour plus d'un. Peut-on alors parler de la nature comme d'un motif important de déplacement touristique? Est-ce que nos forêts extraordinaires attirent les New Yorkais? Que fait-on pour 
intéresser les non-Québécois au Québec? Car il faut bien admettre que ces réussites dont nous parlions tantôt, ces pêcheurs, ces chasseurs, ces skieurs, ces adeptes du plein air et de la promenade en forèt, ce sont presque exclusivement des Québé. cois; les autres font-de même chez eux. Les wétrangersw qui viennent chez nous se limitent aux milieux urbains de Montréal et Québec. Est-ce que nos grands espaces sont encore associés au diminutif kquelques arpents de neiges?... C'est bien beau des "grands espaces" mais comment ceux-ci ont-ils été organisés, aménagés, mis en valeur, publicisés, rendus accessibles, présentés aux visiteurs? Par exemple, nous admettons tous que le SaintLaurent est une ressource fantastique mais est-ce que son potentiel récréatif et touristique a été développé, mis en valeur? Au plan du phénomène physique, nous le qualifions volontiers d'uuniquew; même le célèbre commandant Cousteau est émerveillé... mais au-delà des énoncés de principe, qu'y a-t-il de bien concret outre quelques rares bateaux de croisière, quelques rares marinas ici et la $\mathrm{a}$ C'est presque une désolation que d'avoir une ressource aussi exceptionnelle et de si peu la mettre en valeur, sauf pour le transport maritime et comme dépotoir universel pour trop d'entreprises, de municipalités, de navires,... Est-ce que les Québécois connaissent leur fleuve et estce qu'ils peuvent en parler autrement qu'en critiquant la vitesse de croisière sur les ponts qui l'enjambent? La fierté de ce que l'on a découle d'une bonne connaissance de la ressource et on peut parier que les Québécois en général ont une assez faible connaissance du milieu naturel québécois et ils peuvent donc assez difficilement communiquer leur fierté à l'extérieur! Dans ce contexte, il faut se réjouir de la campagne a La fierté a une ville» que vient de lancer la ville de Montréal pour sensibiliser la population même de la ville à se faire les premiers walliésw de leur ville dans un nouvel effort de promotion. La fierté des Montréalais alliée au Boléro de Ravel, ça ne peut que donner des résultats positifs, peut-être pas autant que des slogans tels al love New Yorkn ou aVirginia is for Lovers", mais il s'agit sûrement d'un geste heureux.

Pour conclure cette partie, on pourrait dire qu'en général, les caractéristiques du milieu naturel n'expliquent pas à elles seules le déplacement touristique mais qu'elles sont souvent un cadre essentiel. Le rocher Percé est intéressant en soi, mais partirait-on de Montréal seulement pour aller prendre une photo de soi devant le rocher Perces? On peut en dire autant du canyon du Colorado ou de la Tour Eiffel. On part pour un ensemble intégré d'attractions et d'activités même si certaines composantes accrochent plus que d'autres. L'expérience touristique, c'est un ensemble de stimuli d'ordres très divers et les qualités du milieu naturel, de I'environnement physique, sont très souvent les mieux cotés dans l'ordre des stimuli; plusieurs auteurs en font foi. Par exemple, analysant les besoins fondamentaux des vacanciers, Just Krippendorf fait une synthèse de plusieurs études sur le sujet: «Premidrement, te touriste veut se debarrasser des multiples sollicitations auxquelles il est soumis au cours de sa vie quotidienne: agitation, bruit, hate. Deuxièmement, il a besoin de sortir de la monotonie, de quelque chose de très different. En troisième lieu, il s'agit de se liberer des pressions quotidiennes. Pour se liberer, l'eloignement physique du cadre habituel est nécessaire. Pour ce faire, nous vovons l'importance primordiale du cadre des vacances. Parmi les facteurs de motivations, les attraits du paysage viennent en tête de 20 points d'intérêt. ${ }^{(4)}$ De son côté, les observations faites par Marc Laplante et un groupe d'étudiants de I'UOAM à partir d'un test de motivation au voyage élaboré selon la théorie de Dean MacCannel| 5 i arrivent à peu près aux mémes résultats: sur 33 propositions auxquelles des répondants étaient invités à exprimer leur accord ou leur désaccord avec chacun des énoncés en les pondérant par un facteur compris entre 0 et 5 , on remarque que l'énoncé qui a obtenu le score le plus élevé est le suivant: «Un voyage est toujours plus intéressant s'il permet de vivre un certain temps dans un paysage naturel différent du sien (dépaysement physique) w. (6) Mais encore une fois, ce paysage intéressant, bien qu'essentiel, n'est qu'une composante de ce qui est recherché, une expérience qui aréfère à quelque chose d'extraordinaire, quelque chose de fort, qui doit atteindre certains points culminants de plaisir, d'émotions (peak experiments). De tels depaysements s'obtiennent plus facilement hors de Venvironnement quotidien. (...) Officiellement, le touriste part pour aller voír des choses exceptionnelles: les paysages, les climats, les musées, les grandes attractions, etc. La promesse de plaisir est attachée a la fréquentation de ces réalités fabuleuses et extraordinaires. Au retour cependant, la mémoire conserve plus longuement et avec plus de précision les observations des modes de vie, des coutumes, des habitudes sociales et culturelles des populations fréquentées. $x^{(7)} \mathrm{Ce}$ qui fait dire à l'auteur qu'en définitive, le touriste part de chez lui dans le but non avoué d'embrasser d'un seul regard son propre mode de vie et donc en cherchant à se mieux comprendre luimême! Le tourisme est une activité complexe, un système où tout est interrelié, et bien malin serait celui qui pourrait dire ce qui rejoint le plus tel vacancier: est-ce le vol en avion, la visite du Louvre, la vue de la tour Eiffel, I'ambiance des Folies Bergères, la promenade en bateau de la Seine, la façon de conduire des Parisiens et leurs inénarrables prises de bec, le piquant de la moutarde dans les sandwichs au jambon, les demoiselles aux petites vertus de la Place Pigalle, les caprices de la température, les gens sympathiques rencontrés, les indispositions intestinales, la nourriture, la faune et la flore particulières, la perte des bagages, les cocasseries linguistiques... etc? Ce touriste est sur-stimulé, exité; les sens sont plus aigus, les perceptions aussi, que dans la vie quotidienne. Le voyageur est dans un monde qui lui donne des compréhensions et visions plus aiguës qu'à l'ordinaire. Le charmant petit lac situé à 20 kilomètres du domicile de notre touriste passera presqu'inaperç, alors qu'il sera emballé et prendra maintes photos d'un lac similaire à 2,000 kilomètres de chez lui, pendant un voyage d'agrément à l'étranger.

\section{Qui se laisse attirer}

par «dame naturew?

Une nouvelle variable mérite d'être ajoutée à la discussion à titre d'elément d'explication supplémentaire. Déjà en 1975, Berry constatait que l'esthétisme paysager, en plus d'être une valeur subjective, faisait partie d'une réalité complexe. II alléguait en effet que l'évaluation de ce critere variait selon le statut socioéconomique, l'âge et la distance de l'observateur face au payage, (8) La plupart des auteurs qui ont abordé cette facette du comportement touristique vont dans le même sens. Outre MacCannell (que nous laissons a Marc Laplantel), nous nous devons de faire part ici du fruit des recherches très élaborées du sociologue Bernard Kalaora sur l'accessibilité aux espaces naturels et particulièrement aux forêts péri-urbaines par les populations citadines. ${ }^{\text {(9) }}$

Selon lui, plusieurs enquêtes européennes font apparaître des constantes qu'il faut absolument prendre en charge quand on propose la nature comme attrait touristique: a) la forêt-loisir est fréquentée différemment selon les classes sociales; b) l'influence du niveau socio-culturel dans la consommation de forêt est encore plus déterminante que le revenu; c) certains perçoivent la forët pour elle même et viennent y chercher le sentiment perdu d'une nature et y retrouver leur identité alors que d'autres ont avec elle une relation instrumentale et la perçoivent par opposition à la vie moderne; d) ces attitudes différentes génèrent des comportements différents: certains voudront peu d'aménagement parce qu'ils rechercheront l'air pur, l'évasion, le repos, les autres, appartenant à la civilisation urbaine, voudront une forêt commode, confortable, d'accès facile, e) la sur-utilisation de la forêt par les catégories sociales les plus favorisées est paradoxale; raisonnant en termes de besoins, on aurait pu exactement supposer le contraire. 
Bernard Kalaora s'interroge sur les conditions qui aboutissent a la production de privilèges dans le cas de ce bien dont la consommation est formellement ouverte à tous mais réellement réservée à quelques-uns. II tente ensuite de répondre de la façon suivante.

Wous sommes donc amené à faire Ihypothèse que la forêt est un bien symbolique qui, comme l"aeuvre d'art ne peut exercer preférentiellement d'attraction que sur les personnes deja en possession de la culture. Des pratiques de plein air telles que la marche, la promenade, la randonnée exigent de la part du visiteur la capacité a reconnaittre et à différencier les paysages selon leur qualite esthétique mais aussi l'aptitude a maitriser l'espace. // existe certainement une relation entre l'approbation de l'espace et /'univers spatial du sujet. Lorsque les contraintes spatiales (univers morcele de travail, de /habitat, de la consommation) sont trop nombreuses, I'individu ne peut prendre aucune initiative et vit la quotidienneté...p (p. 95)

"La forêt, nous avons essayé de le montrer, n'est pas I'antithese de la ville, un lieu libéré des contraintes qui d́chapperait aux determinismes sociaux. On vient certes en forêt pour $y$ renouveler sa force de travail, mais on y transporte aussi des valeurs, des normes, des attitudes et des comportements qui sont ceux-la même que crée l'aménagement social de la ville. (...) L'aménagement inscrit dans l'espace la division sociale en classes antagonistes par une delimitation du territoire selon les besoins spécifiques de tel ou tel groupe social, reproduisant ainsi la ségrégation spatiale.w (p. 283 et 285)

Les conclusions auxquelles arrivent Kalaora ne vont pas sans rappeler des questions que nous posions récemment dans Téoros (Vol. 1, no 3) même sur le tourisme social, en cherchant à comprendre, par exemple, pourquoi les utilisateurs de services comme l'hébergement à la ferme, originellement orientés vers les familles moins bien nanties, ne retenaient l'intérôt en fait que de la clientèle favorisée au plan économique et social. Le sociologue dont nous rapportons ici la pensée répondrait sans doute que les classes défavorisées culturellement sont incapables de développer un intérêt quelconque pour ce type de vacances, pour le milieu rural et naturel en général, de sorte que même si on leur offrait ces vacances gratuitement, elles seraient peu intéressées. Sans prétendre qu'un diplôme collégial ou universitaire est nécessaire pour apprécier l'arôme d'une épinette, le vol d'un goéland à ceinture fléchée ou la culture des champignons comestibles, il est probable en effet que la préparation culturelle d'un individu détermine dans une bonne mesure l'inté- rêt qu'il est susceptible de porter au milieu naturel. II serait intéressant de connaître le profil des gens qui fréquentent les parcs provinciaux, par exemple, ou de ceux qui vont apprécier le mont Albert ou visiter le Jardin Botanique; sans doute pourrionsnous y vérifier les théories exposées par Kalaora dans une bonne mesure.

L'appel de la nature et le touriste

Si la réalité est telle, l'éducation du public en général serait le meilleur outil pour favoriser l'attrait que peut représenter le milieu naturel auprès des vacanciers. $\mathrm{Or}_{\text {r }}$ on assiste depuis bientôt une décennie à une espèce d'engouement pour la nature, et singulièrement pour la forêt qui auraient soudainement des vertus magiques. Autrefois dangereuse, obstacle au progrès et à la croissance, elle est devenue aujourd'hui le salut de la ville (la ceinture verte est une protection de la ville contre elle-même). Tous les regards convergent vers elle et on assiste à une véritable mythification de la nature et surtout de I'arbre, de l'air, du vert. En Allemagne de I'Ouest on vient même d'élire des députés «Verts" et «Vertes» (?). On voit naitre des partis politiques ácologiques»... Les effets bénéfiques de la nature, de la verdure, s'ils sont désirés et si tous devraient $y$ avoir accès, sont dans la ville des biens rares et cette rareté valorise les zones où ils sont présents. ( $\mathrm{A}$ l'inverse, on peut se demander si la surabondance d'espaces verts sur l'ensemble du territoire québécois ne lui fait pas perdre une grande partie de sa valeur aux yeux des Québécois, peu soucieux d'en prendre grand soin car il y en a tellement?) Le vert et son attribut l'air se monnayent désormais en milieu urbain. ${ }^{(10)}$

Ce cheminement nous entraîne vers les notions d'authenticité recherchée par les vacanciers et de sacralisation du produit. Plusieurs études le démontrent: le touriste est souvent soupçonneux; il craint de se faire aemplirs par des belles paroles, du clinquant, de la poudre aux yeux. C'est encore MacCannell qui écrit que a/instabilité et la superficialité de la société moderne a engendré au niveau des valeurs l'idée que le réel et l'authentique sont ailleurs, dans d'autres perriodes historiques, dans d'autres cuitures ot dans d'autres styles de vie plus simples et plus purs. C'est un des fondements de la conscience unifiante de notre époque. Que va-t-on voir au Village québécois d'antan, de Drummondville? Sur les Plaines d'Abraham? Dans le Vieux Montréal? A la campagne? Le touriste ne veut pas se faire passer du faux, il faut lui prouver l'authenticité des choses, même si ces choses ne sont plus que des ruines. On se passionne pour une queue de dinosaure, pour ces musées qui raniment le passé, pour les peuples primitifs qui avaient une vie champêtre, rurale, paisible, plus simple et plus pure, pour ces petits villages, coins perdus loin des grandes villes et donc mieux conservés, et donc plus vrais et moins artificiels. L'intére̊t grandit pour le uwilderness\%, pour la vie sauvage, pour l'écologie, pour les milieux vierges, pour le folklore et les fêtes populaires; pour l'artisanat, fait à la main, donc plus vrai lque les répliques du rocher Percé «made in Taïwanw! ou que de manger des crevettes de la mer du Nord quand on visite Matane!)

Aménager l'état vierge de la nature! L'attraction touristique implique l'intervention humaine. II y a 10 ans, la gorge de Coaticook était un dépotoir à ciel ouvert; aujourd'hui, après l'avoir nettoyée, construit des sentiers et des passerelles, expliqué les phénomènes géologiques impliqués, installé des bancs et divers autres services, 40,000 personnes s'y arrêtent chaque été. Bien sûr, ce degré d'intervention humaine est très variable. A Disney World, on est parti d'un marécage hostile à la présence humaine et on a construit un gigantesque complexe récréatif de $\mathrm{A}$ à $\mathrm{Z}$; par contre, la transformation des chutes Niagara ou Montmorency en attraits a surtout été faite en l'annonçant, en l'illuminant et en organisant des visites. C'est ca la sacralisation du produit; c'est investir un site d'un certain sens de sacré, qui va le rendre attrayant. Encore là, cette force attractive peut être développée à différents niveaux selon l'objet soumis au processus. Par exemple, le grand Canyon (ou les Plaines d'Abraham) n'a pas tellement besoin d'être sacralisé on n'a qu'à mettre des flèches pour permettre aux visiteurs de s'orienter (quoique la flecche en question est déjà un usignew, une reconnaissance de sacralisation). L'événement est suffisamment grand en luimême.

Chez nous, que fait-on pour sacraliser le produit? Si on demandait aux. Québécois d'identifier 25 sites ou manifestations touristiques, il est probable qu'ils auraient beaucoup plus de facilité a le faire aux Etats-Unis ou en France qu'au Québec mềme. Est-ce par manque de connaissances? manque de fierté? manque d'attraits? manque de motivation? Serait-ce normal que les Québécois connaissent mieux le produit américain que leur propre patrimoine?... ou est-il attirant parce qu'ailleurs? Dans ce cas, ça devrait aussi être vrai pour les Américains.

Le climat et les saisons: des ressources à gérer

Bien sûr, on dira qu'il y a la température, aussi incertaine le 16 janvier que le 7 juillet, et qu'on tire peu avantage des saisons. "The dominant undeveloped resource factor is climate. Participation in most outdoor activities is affected to some extent by weather patterns.... ${ }^{(12)}$ 
On doit admettre qu'exception faite du Grand Nord québécois, notre climat en général est peu fiable et pleins de surprises; il ne se classe sûrement pas parmi les conditions idéales:

un optimal recreation climate can be defined as one that will permit participation in confort for a maximum number of days each year in walking, playing games, or sitting out-of-doors relaxing in the sun... On that basis, Mediterranean chmate have the most favorable year-round combination of moderate temperatures and clear skies. The remaining humid warm temperatures climates come next, with reasonably mild temperatures but less sunshine. The tropical and dry climates tie for third place; heat, humidity, and heavy rains are the disadvantages of the former, heat, aridity and cold are the shortcomings of the latter. Snow and ice climates occupy fifth and sixth place respectively. $w^{\text {(13) }}$

Notre climat et nos saisons sont des composantes importantes de notre milieu naturel et s'il faut en croire l'échelle climatique proposée par Chubb, notre situation ne serait pas très favorable. C'est un fait que les sauts d'humeur de notre climat ont maintes fois causé de sérieux désagréments à nos vacanciers c'est d'ailleurs aussi le cas a Old Orchard, à Miami, ả Londres ou à Val d'isère. Notre climat capricieux pose des problèmes mais l'alternance des saisons qu'il permet vaut bien ce prix, encore que l'on puisse se prémunir contre ces désagréments en aménageant des complexes intégrés de vacances quil ne sont pas basés sur la pratique d'une seule activité, comme c'est encore trop souvent le cas ici. Ainsi, il est certain qu'en l'absence de neige pendant quelques jours, des stations comme I'Estérel ou Mont-Tremblant seront moins désorganisées qu'Orford qui n'offre pas d'autres alternatives.

W... many early Appalachian Mountain Resorts were developed, at least partly. because of the climatic relief afforded at those locations. In winter, higher elevations and lower temperatures result in the snow accumulations so attractive to winter-sports enthusiasts. (...) Recreation is dependant on vegetation in many ways. In some cases, it is the primary component. California's giant trees, the sequolas and the redwoods, are rated as the US fifth most popular bioliogical or physical tourist attraction. In the autumn, the changing colors of various tree species create a third tourist season for the New England States. $y^{(14)}$

Donc, s'il est vrai que le climat pose une contrainte, il faut admettre qu'il a aussi des avantages et des bons côtés. D'ailleurs, ce climat, ces saisons, cette végéta- tion, cette nature, ce réseau hydrographique, cette faune, c'est en fait tout ça qui fait que notre milieu naturel est unique, spécifique au Québec. Mais, que faisonsnous pour mettre ce fantastique patrimoine en valeur, pour le rendre attrayant aux yeux des visiteurs? Derriere cette nature, cette ressource naturelle, il doit bien y avoir une théorie d'attraction quelconque, un concept de mise en valeur mis au point par les promoteurs du produit? La ressource est là! Comment maintenant l'organiser en apackagew ayant suffisamment de choses pour motiver un départ pour venir consommer ce produit en tant qu'attrait? Est-ce que le gouvernement du Québec s'intéresse au tourisme autrement que pour régler ses problèmes de balance des palements deficitaire? On veut que les Québécois voyagent au Québec mais les efforts pour rendre attirant ce pays sont assez limites; on prendrait donc pour acquis qu'il est normal et naturel pour les Québécois de prendre leurs vacances chez eux, d'autant plus que c'est dans ala belle provincen qu'ils ont le plus ude parents ou d'amiss! II $n^{\prime}$ est pas suffisant de dire qu'on a de beaux lacs, de belles montagnes, de l'essence moins chère que sur la lune, qu'on a de grands espaces. C'est quoi un grand espace?

Peut-on dire qu'un des problèmes majeurs que rencontre la mise en valeur de notre patrimoine naturel et la sacralisation de ses sites, c'est que ce territoire est trop grand pour nos movens, que la tâche est colossale et qu'on doit $y$ aller petit peu par petit peu? Si cette explication est justifiable, il serait alors injuste de comparer notre niveau de développement touristique avec celui des Américains, comme on le fait souvent, car ils sont 250 millions à pouvoir mettre la main à la pâte. Pourquoi les quelques centaines de milliers de Québécois qui traversent les Montagnes Blanches en route vers les côtes Atlantiques, ont-ils tendance à faire des comparaisons? Devant ces paysages, au Québec, on dira: " $\mathrm{C}^{\prime}$ est beau, c'est grand, c'est à voir! $n$ Devant les mêmes paysages, I'Américain construira une halte routière scénique et des petits sentiers serpenteront ça et là pour permettre de bien saisir toute la richesse du site, lequel sera d'ailleurs baptise (Rising Sun Falls). Sur des pancartes en bois grave, on informe: aCet arbre est un érable de Virginie; arrivé à maturité, il peut atteindre $X$ mètres de hauteur et $Y \mathrm{~cm}$ de circonférence. Ce sont les indiens Mohawks qui ont appris à en tirer la sève sucrée... Sa graine a l'air de ceci et ses feuilles à cela. Cet arbre pousse de préférence dans un sol argileux et.... Et lä-bas, cette autre pancarte: aCe cours d'eau a mis plus de trois millions d'années à se frayer un passage dans cette roche surtout composée de calcaire et de schistes argileux laisses sur place par la fonte rapide du glacier labradorien qui recouvrait toute cette région, il y a encore 33,000 ans... $w$ Bref, on explique le paysage, on le démystifie, on le rend sympathique mais aussi spécial. Ça aussi, c'est de la sacralisation. De plus, ces pancartes, ces affiches, ces umarqueurs sont des témoignages d'experts qui authentifient les informations données. Combien de milliers de Québécois arrêtent quelques moments à ces haltes routières, précurseurs de bien mieux encore?

\section{La nature: un produit exportable?}

Au cours de 1982, le MICT a fait effectuer une étude de grande envergure aux ÉtatsUnis afin de mieux connaître notre principal marché extérieur. Le but principal de cette recherche était de adéterminer la perception du produit touristique québé. cois qu'ont les Américains ayant fait un voyage d'agrément (au Québec) au cours des 24 derniers mois. 3 Pas moins de 8,081 appels téléphoniques ont été effectués et 2,250 interviews ont été complétées, par la firme Davidson, Peterson et Associés Inc. de New York, soit 375 pour chacune des villes suivantes: Boston, Chicago, Hartford, New York, Providence et Washington, D.C. (15). Une étude du même type a été récemment réalisée par la firme de sondage ICOP pour le MICT et porte sur la perception qu'ont les Québécois du produit québécois. Malheureusement, les résultats de cette dernière recherche sont pour le moment maintenus confidentiels et en dernière heure, wons apprenait que les sous-ministres en étaient à la phase aépurations! Nous allons donc revenir à I'étude portant sur la clientẻle américaine pour tenter de savoir si la nature, le milieu physique québécois, est, à leurs yeux, une attraction suffisamment forte pour les inciter à venir visiter ala province qui se souvient» (qu'il fût un jour, pas lointain du tout, où ces Américains venaient en bien plus grand nombre).

Étant donné que le MICT a effectué cette étude dans une perspective de umise en marchê du produit touristique québécoiss. aussi bien que ade développement de ce dit produitw, il sera intéressant de voir si la perception qu'a la clientèle de ces 6 marchés du produit québécois correspond a ses attentes. Quelles sont ces attentes? Qu'est-ce qui apparaît particulièrement wessentiels à ces Américains lors d'un déplacement touristique.

En voyage de vacances, les Américains consultés recherchent, par ordre d'importance, le confort de l'hébergement, des lieux reposants, le soleil, le sable et la mer. Viennent ensuite l'environnement agréable pour les promenades à pied, les prix raisonnables, les gens du milieu et la vie nocturne, le magasinage et finalement, la cuisine régionale dans de petits restaurants typiques. $39 \%$ des répondants trou- 
vent cabsolument essentiels le confort des établissements alors que $16 \%$ ont la mêrme exigence à propos de la cuisine régionale ${ }^{\mid 16}$,

Si nous présentons maintenant â ces mểmes personnes divers visages du Québec, voici comment elles les perçoivent: a) les traits suivants, par ordre d'importance, sont reconnus par plus de $50 \%$ des répondants: le paysage et l'environnement agréables à regarder, la culture différente, l'intérêt pour des vacances en famille, en couple, une destination pour le ski et les activités hivernales; b) l'image du Québec couvert de beaux lacs est perçue par $45 \%$ des Américains et $37 \%$ reconnaissent que notre température est propice aux vacances; c) I'accord sur les traits suivants varie de $33 \%$ à $24 \%$ : la qualité de la signalisation touristique, I'information touristique, le coût raisonnable des services, l'état des routes et les moyens de transport à l'intérieur de la Belle Province.

Bien sûr, toutes ces données sont tellement riches et instructives qu'il faudrait plusieurs dizaines de pages pour en bien saisir toute la substance, ce qui ne peut être fait ici présentement. Nous retiendrons tout de même que la clientèle de notre principal marché (hors Québec) perçoit très positivement notre milieu naturel mais elle semble lui attribuer de sérieuses lacunes d'accessibilité, sans compter qu'elle estime que le produit, compte tenu de al'attirances de son unicité, est passablement dispendieux. D'autre part, les Américains vivant dans les grandes agglomérations proches du Québec ne semblent pas rechercher d'une façon spéciale une région de vacances où l'attrait principal serait les abeaux paysagess, exception faite des stations balnéaires où on retrouve les 3,4 ou 5 «Sw. Donc, si l'Américain perçoit le Québec comme ayant surtout un produit smilieu naturelw à lui offrir, qu'il ne recherche pas particulièrement une expérience touristique axée sur la nature, surtout si cette nature n'est pas à ses yeux exceptionnelle, tout à fait unique, qu'il peut trouver un produit semblable chez lui, plus près, cela peut sans doute expliquer dans une certaine mesure pourquoi cet Américain délaisse de plus en plus le Québec depuis 10 ou 15 ans.

Dans son chapitre sur la demande, Marc Laplante n'hésite pas à écrire: vLe désir le plus courant a lorigine du projet de voyage de vacances est la recherche de I'expérience stimulante, notamment de fextraordinaire, de l'unique et du jamais $v u^{\prime}, y^{(17)}$ Se peut-il que le milieu naturel québécois ne réponde pas à cette exigence pour la majorité de cette clientèle, malgré nos Laurentides, malgré Anticosti, les Îles de Minguan ou de la Madeleine, malgré l'íle d'Orléans, la Gaspésie et I'Estrie, malgré nos troupeaux de caribous ou les safaris-photos aux baleines de l'estuaire du Saint-Laurent? Quelle est la perception des Québécois sur ces mêmes produits? Faut-il plutôt conclure que l'essentiel reste à faire pour que la réalité de la nature québécoise devienne à la fois une fierté pour les nationaux et un produit touristique exportable?

\section{Conclusion}

Concrètement, ou cela nous conduit-il? Sans doute à un désir de tirer plus avantage de cette immense richesse qu'est le milieu naturel québécois, à le mettre en valeur, à le faire connaître sous ses angles les plus originaux et donc, à en faire ainsi un produit recherché.

Pour y arriver, il faudrait, à court terme, donner a tous les promoteurs du produit touristique québécois a tous les niveaux des ubriefingsw intensifs sur le potentiel des ressources du milieu naturel comme éléments des produits touristiques. Pour cela, il faudrait repenser les modalités de la concertation dont on aime tant parler en tourisme. Des sommets, comme celui de Sherbrooke en 1978, ont tenté de faire se concerter les intervenants mais les participants n'ont pu que déplorer notre sous-développement sans apporter des éclairages nouveaux sur ses causes. Une autre sorte de concertation s'impose, celle qui réunira autour d'une même table des créateurs de produits touristiques et des spécialistes du milieu qu'on veut offrir aux futurs visiteurs. Par exemple, dans le cas qui nous occupe ici, une bonne session d'études regroupant des botanistes, des naturalistes, des biologistes, des géographes, des écologistes, des hornithologistes, des spéléologues, des alpinistes, des navigateurs, etc. et des créateurs de forfailts, d'itinéraires, des auteurs de brochures touristiques, des responsables de la promotion et de l'information enrichirait les uns et les autres. Car, au Québec, la nature et les richesses qui s'y cachent sont connues et même bien connues; simplement ceux qui les connaissent ne s'occupent pas de tourisme et ceux qui attendent tout du tourisme ne les connaissent pas.

Des concertations de ce genre ${ }^{(18)}$ ne sont ni difficiles, ni coûteuses. Les projets qui en résulteront pourront se présenter par étapes, visant d'abord les amenagements ou ré-aménagements simples et peu co0teux (signalisation, informations supplémentaires dans les kiosques, correction des parcours, etc.). Des programmes de 4-5 ans de mise en valeur des produits touristiques peuvent aussi se concevoir par des concertations comme celles que nous proposons ici.

A plus long terme - mais avant I'an 2000
- il faudra aussi que les générations futures de Québécois et de Québécoises (tant pis pour les irrécupérables d'aujourd'hui!) s'imprègnent de leur milieu naturel et apprennent a vivre en symbiose avec lui. Si les visités I'ignorent ou la méprisent, comment faire valoir notre belle nature à des étrangers de passage? Alors, professeurs d'histoire et de géographie, maîtres des sciences naturelles et biologiques, savants professeurs d'écologie revoyez vos programmes et vos pédagogies, arpentez le Qúbec avec vos étudiants produisez cent vidéos par région pour la T.V., etc. mais faites en sorte que les Québécois connaissent leur inimaginable pays: a cette condition seulement, ils pourront vraiment I'aimer. Alors, les quelques millions de Québécois qui partent en vacances chaque annee seront autant de bons agents de promotion pour le Québec. Et, en plus, ceux et celles qui prendront al'tour du Québecn, n'agiront plus ainsi parce que c'est moins cher mais parce que cette destination les attire. $f$

\section{Références}

(1) NADEAU, Rager at colliborateurs, Le Teurisme: aspects theoriques et pratiques au Qubbac, Association Technique du Tourisme (ATT), Sodilis Editeur, Montestal, 1982,206 pages.

(2) FAPEL, Les wilgiotews dquilibert le budget de 126 mumicipulids, article paru dans La Presse. Montrobl, 18 avril 1963. IFAPEL: Foderation des Associations pour a Protection de I'Emvironnement des Lacs. I

(3) MLCP, Plein air - Infroduction; Exploration souterraine, tandonnee pedeatre, randonnde al woile, randonnee equestre, collection Plain Air, Ministère du Loisir, de la Chasse et de la Pêche, Quebec, 1983, 35 pagers.

(4) KRIPPENDOAF, J., Las divorours do paysagas: le tourisme doit-il detruire les sites qui le font vivre? 24 heures Imprimeries, Lausanne, 1977, 157 pages.

(5) Mae CANWEL, Dem, The Tourist: A New Theory of the Leisure Class, New York, Shocken Books, 1976 .

(6) LAPLANTE, Marc, La demande tourlstique au Quebec, in NADEAU, Roger et collaborateurs, 1982, op. cit. p. 96.

(7) Ibid, p. 86 .

(8) BERRY, D., Landscape Aosthetics and Enviranmental Planning: A Critique of Underlying Premises, Regionsl Science, Fesearch Institute, Discussion Paper Series No 65, Philadelphia, 1975, 30 pages.

(9) KALAORA, Dernard, Le musde vert - ou le tourisme on forbt: Naissence et df́veloppement d̛ un laisir untain: le cas de la foret de Fontainebleau, Editions Anthropos, Paris, 1901,304 pages.

(10) Ibid, p. $\boldsymbol{2 9 1 .}$

111) MAC CANNEL, op of

(12) CHUBE, Michael 6 Hally R.; One Third of Our Time? An Introduction to Pecreation Behavior and Ressources: John Wisy 4 Sons Inc., New York, 1981, 742 pages (p. 153).

(13) Ibid.. p. 307

(14) Ibid., p. 317 et 319.

(15) CLUZEAU, P.: THIFFAULT, G. Sondage sur la perception du produit touristique quebdecis qu'ont los Ambricains ayant fait un voyage d'agrament as cours des 24 derniars mois ot sur le niveau de pand. tration de la campagne publicitaire U.S. dte 1981 MiCT; Analyse et prógrammation; Direction getnérale du Tourisme, Quabec, awil $1962,360 \mathrm{p}$.

116) CLUZEAU, P.; THIFFAULT, G.; 1982, 09, ett, p. 139.

[17] LAPLANTE, Marc; 1982, 00, cit. p. 86

(19) Concevables pour toutes les grandes compcsantes du produit touristique globel quebescois et non uniquement pour la nature, II va sans dire. 